\title{
Robust Blind Beamforming in Impulse Noise Environments
}

\author{
Zheng Liu ${ }^{1}$,Peng Liu ${ }^{2}$,Jiyan Huang ${ }^{1}$,Daoxin $\mathrm{Li}^{3}$, Hongyan Zhang $^{1}$, Biwen Wang ${ }^{1}$ \\ ${ }^{1}$ University of Electronic Science and Technology of china (UESTC), Chengdu, \\ China(huangjiyan@uestc.edu.cn) \\ ${ }^{2}$ Space Star Technology Co., Ltd. and State Key Laboratory of Space-Ground Integrated Information \\ Technology, Beijing, China
}

${ }^{3}$ North engineering Co.,LTD of the electrification bureau group, crcc, Taiyuan, China

Keywords: Blind beamforming, Cyclostationarity,Factional lower order matrix, $\alpha$ stable noise environment.

Abstract: Compared with the conventional adaptive spatial filter algorithm, blind beamingforming algorithm is endowed with the capacity to protect desired signal, suppress interferences and reduce noise without the information about arrival direction of desired signal, thus leading to the wide application in many fields. In previous studies, the background noise was assumed to be gaussian noise distributed. In case the ambient noise is non-gaoussian noise, the performance of classic blind beamforming algorithm known as spectral self-coherent restoral method would experience a rapid deterioration. Based on the fractional lower order matrix and robust estimation theory, this paper provides a solution to the problem of blind adaptive beamforming in $\alpha$ stable noise environment, and the proposed method can be verified by the simulation results.

\section{INTRODUCTION}

With the rapid development of adaptive filter theory and algorithm, the adaptive filter technology has achieved an extensive application with increasing attention in recent decades [1-2]. It has been widely used in radar, seismology, radio astronomy, medical imaging, speech processing and wireless communications [3-5]. Through in-depth studying, researchers put forward a great deal of non-blind or blind adaptive beamforming algorithms, such as maximum signal-to-noise ratio beamforming, minimun noise power beamshaper, and minimum variance distortionless response [6]. Called non-blind adaptive beamforming algorithms, these algorithms require the knowledge of directions of the desired signal or training information, while the blind adaptive beamforming takes advantage of the cyclostationarity of signals to restore the desired signal. As for the performance of classic blind beamforming algorithm known as the spectral self-coherent restoral (SCORE) method, it retains consistence with the performance of MVDR algorithm when the number of snapshots goes to infinity. Moreover, it should be noted that SCORE algorithm is put forward based on the assumption of gaussian noise. However, this kind of assumption is rarely good enough owing to the interference of wireless channels, atmospheric, and receiver in a real life situation. Unfortunately, due to the divergent sample correlation matrix under the condition of the non-gaussian noise, the performance of conventional SCORE algorithm has experienced a sharp decline.

To suppress non-gaussian noise, a method based on fractional lower order matrix in [7] is proposed for non-blind beamforming technique. In this paper, a novel approach is proposed to suppress the $\alpha$ stable noise for restoring the performance of SCORE algorithm. Main contributions of this paper are listed as follows:(1)Fractional lower order matrix is extended from non-blind beamforming [7] to blind beamforming.(2)Besides fractional lower order matrix, M-estimate function is also used in the proposed method for mitigating non-gaussian noise. 


\section{BACKGROUND KNOWLEDGE}

I.Conventional SCORE Algorithm

It is assumed that there is a uniform linear array with $M$ sensors receiving $N$ narrow-band signals containing one desired signal and $N-1$ interference signals emitted from the far field. The $M \times 1$ vector of receiving signal can be represented as:

$$
\mathbf{x}_{k}=\mathbf{a}(\theta) \mathbf{s}_{k}+\mathbf{n}_{k}
$$

where $\mathrm{k}$ is the sampling time, $\mathbf{s}_{k}$ is the desired signal and interference from respective direction angle $\theta$, $\mathbf{a}(\theta)$ is a $M \times 1$ steering vector at direction angle $\theta$, and $\mathbf{n}_{k}$ is the $M \times 1$ vector of noise (gaussian or non-gaussian ). Through solving the optimal or suboptimal weight vector $\mathbf{w}$ without knowing priori information about any relevant desired signals or interference, we can obtain the desired signal $\mathbf{s}_{k}$ from receiving signal $\mathbf{x}_{k}$ by SCORE algorithm. The output of the beamforming is given by:

$$
\mathbf{y}=\mathbf{w}^{H} \mathbf{x}
$$

The cost function of SCORE algorithm can be written as:

$$
\max _{\mathbf{w}, \mathbf{c}} \frac{\left|\mathbf{w}^{H} \mathbf{R}_{\mathrm{xu}} \mathbf{c}\right|^{2}}{\mathbf{w}^{H} \mathbf{R}_{\mathrm{xx}} \mathbf{w c} \mathbf{c}^{H} \mathbf{R}_{\mathrm{uu}} \mathbf{c}}
$$

where $\mathbf{u}(k)=\mathbf{x}^{*}(k+\tau) \exp (j 2 \pi \alpha k)$ is the referent signal. $\mathbf{R}_{\mathbf{x u}}=\left\langle\mathbf{x}(k) \mathbf{u}^{H}(k)\right\rangle_{k}, \mathbf{R}_{\mathbf{x x}}=\left\langle\mathbf{x}(k) \mathbf{x}^{H}(k)\right\rangle_{k}, \quad \mathbf{R}_{\mathbf{u u}}=\left\langle\mathbf{u}(k) \mathbf{u}^{H}(k)\right\rangle_{k}$, in which $\mathrm{k}$ is the number of snapshots, and $\mathbf{c}$ means the referent vector.

$$
\mathbf{R}_{\mathrm{ss}}(i, j)=\left\langle\mathbf{s}_{i}(k) \mathbf{s}_{j}{ }^{H}(k)\right\rangle_{K}=\frac{1}{K} \sum_{k=1}^{K} \mathbf{s}_{i}(k) \mathbf{s}_{j}{ }^{*}(k)
$$

II.The characteristics of $\alpha$ stable distribution

The definition of $\alpha$ stable noise model based on characteristic function is given by:

$$
\varphi(t)=\left\{\begin{array}{l}
\exp \left\{j \mu \mu-\gamma|t|^{\alpha}\left[1+j \beta \beta \operatorname{sig}(t) \tan \left(\frac{\alpha \pi}{2}\right)\right]\right\}, \text { if } \alpha \neq 1 \\
\exp \left\{j \mu \mu-\gamma|t|^{\alpha}\left[1+j \beta \beta \operatorname{sig}(t) \frac{2}{\pi} \log |t|\right]\right\}, \text { if } \alpha=1
\end{array}\right.
$$

where $\alpha$ is features exponent, which determines the extent of noise. $\beta$ is symmetry parameter, which determines the degree of distorted distribution. Moreover, $\gamma$ is scale parameter, which determines the degree of stable variable deviated from the mean, and ${ }^{\mu}$ is positional parameter which reflects the offset of probability density function in $\mathrm{X}$-axis.

The statistic matrix of signal contains wealth of information about the characteristics of signal. For the conventional methods, typically second-order matrix or high-order matrix is utilized. If the signal or the noise characteristic index satisfies $0<\alpha<2$, its higher-order statistics and second-order statistics would not exist, with $\alpha$ stable noise being one of the representatives.

The second-order matrix of random variable $\mathrm{X}$ is typically defined as $E\left[X^{2}\right]$. For the $\alpha$ stable distribution random variable, there is only the existence of fractional order matrix, which can be represented by the following formulas:

$$
\begin{array}{cl}
\text { If } 0<\alpha<2 \text {, then: } & E\left[|X|^{p}\right]=\infty, \quad p \geq \alpha \quad E\left[|X|^{p}\right]<\infty, \quad 0 \leq p<\alpha \\
\text { If } \alpha=2 \text {, then: } & E\left[|X|^{p}\right]<\infty, \quad p \geq 0
\end{array}
$$

In this case, it can be seen from formula (3) that the SCORE algorithm based on higher-order statistics or second-order statistics cannot work effectively. Moreover, there would be the emergence of obvious deterioration, which even leads to erroneous results. Thus, the fractional lower order matrix (FLOM) or fractional lower order statistics (FLOS) has become an important way of signal processing under the condition of signal or noise with non-gaussian noise stable distribution. 


\section{ROBUST SCORE BEAMINGFORMING ALGORITHM}

Before the use of the fractional lower order matrix to improve the algorithm, in this paper, the following method is employed to process the received signal.

An M-estimate function is chosen as follows:

$$
\rho\left(x_{i}(k)\right)=\left\{\begin{array}{c}
x_{i}(k)\left|x_{i}(k)\right| \leq \beta \\
0 \text { otherelse }
\end{array}\right.
$$

where $\beta$ is the cutoff value; when the received signal influenced by $\alpha$ stable noise is much larger than other sampling value (also larger than the cutoff value $\beta$ ), $\rho(\bullet)$ would limit the effect of $\alpha$ stable noise.

The choice of the cutoff value $\beta$ in (7) plays an important role in the performance of the robust channel estimator. It can be seen from (1) that the $\mathbf{n}_{k}$ is $\alpha$ stable noise. The probability of $z_{n}$ greater than the cutoff value $\beta$ is given by:

$$
\theta=P\left\{\left|z_{n}\right|>\beta\right\}=\operatorname{erfc}\left(\beta /\left(\sqrt{2} \sigma_{z}\right)\right)
$$

where $\sigma_{z}$ is the estimate standard deviation of the "impulsive free" error, and

$$
\operatorname{erfc}(x)=\frac{2}{\sqrt{\pi}} \int_{x}^{\infty} e^{-x^{2}} d x
$$

is the complementary error function. For the given percentages of impulsive noise $\operatorname{Pr}\left(b_{n}=1\right)=p$, the value of $\beta$ can be determined with the formula (8).However, the probability of impulsive noise is generally unknown in realistic situation. Therefore, a robust method has to be developed to estimate $\beta$. It can be seen from (8) that $\beta$ depends on $\theta$ and $\sigma_{z}$, the effect of which can be described by the median absolute deviation from the median of $z_{n}$

$$
\widehat{s}=\operatorname{Med}\left\{|| z_{n}\left|-\operatorname{Med}\left(\left|z_{n}\right|\right)\right|\right\}
$$

Thus, the cutoff value $\beta$ can be approximately determined by

$$
\beta=N \widehat{s}
$$

the fractional lower order moment(FLOM) has been successfully applied in MVDR algorithm[7], It is defined as:

$$
\begin{aligned}
& \mathbf{R}_{F L O M}=\left\langle\mathbf{x}(k)\left[\mathbf{x}^{H}(k+\tau)\right]^{(p-1\rangle}\right\rangle_{k} \\
& =\left\langle\mathbf{x}(k)|\mathbf{x}(k+\tau)|^{p-1} \operatorname{sgn}\left[\mathbf{x}^{H}(k+\tau)\right]\right\rangle_{k}
\end{aligned}
$$

Easy to see from the above formula,due to the non -symmetry of $\mathbf{R}_{\mathrm{xx}}^{(p)}$, the SCORE algorithm has poor performance. In particular,when $\alpha<1$, the algorithm fails.Therefore, this paper takes the following approach:

Complex random variables $\mathrm{X}$ is defined as follows:

$$
x^{\langle p\rangle}=\|x\|_{\mathrm{p}}^{\mathrm{p}-1}=|x|^{\mathrm{p}-1} x, 0 \leq \mathrm{p} \leq 1
$$

For $\mathrm{L}$ dimensional vector $\mathrm{x}$, it can be defined as:

$$
\mathbf{x}^{\langle p\rangle}=\left[x_{1}^{\langle p\rangle} \ldots x_{L}^{\langle p\rangle}\right]
$$

By converting $\mathrm{x}$ into polar form, we can get $\mathbf{x}=\mathbf{r e}^{\mathbf{j} \theta}$. Similarly, there is $\mathbf{x}^{\langle p\rangle}=\mathbf{r}^{p} \mathbf{e}^{\mathbf{j} \theta}$. Compared with the former, $\mathbf{x}^{\langle p\rangle(p<\alpha / 2)}$ just changes the information about amplitude, while the information about phase 
remains unchanged. In this paper, under the condition of stable distribution, the fractional lower order matrix and fractional lower order cyclic matrix are applied to the SCORE algorithm.

The fractional lower order covariance(FLOC) is:

$$
\begin{aligned}
& \left.\mathbf{R}_{\mathbf{x u}}^{(p)}(\tau)=\langle\mathbf{x}(k))^{\left\langle\frac{p}{2}\right\rangle}\left[\mathbf{u}^{H}(k+\tau)\right]^{\left\langle\frac{p}{2}\right\rangle}\right\rangle_{k} \\
& =\left[R_{x, u_{j}}^{(p)}(\tau)\right]
\end{aligned}
$$

where $\mathbf{x}(k)=\left[\mathbf{x}_{1}(k), \mathbf{x}_{2}(k), \ldots, \mathbf{x}_{L}(k)\right]^{T}, \mathbf{u}(k)=\left[u_{1}(k), u_{2}(k), \ldots, u_{L}(k)\right]^{T}$, both of them are vectors of length $L,\langle\rangle_{k}$ is the average of time, $p$ is the fractional lower order parameter, $0<p<\alpha$.

The element of ith row and jth column in the matrix is:

$$
R_{x_{i} u_{j}}^{(p)}(\tau)=\left\langle x_{i}(k)\left\langle\frac{p}{2}\right\rangle\left[u_{j}^{*}(k+\tau)\right]^{\left(\frac{p}{2}\right)}\right\rangle_{k}
$$

Similarly, the proprosed the fractional lower order cyclic covariance (FLOCC) is shown as:

$$
\begin{aligned}
& \left.\mathbf{R}_{\mathrm{xu}}^{(p)}(\varepsilon ; \tau)=\langle\mathbf{x}(k))^{\left(\frac{p}{2}\right)}\left[\mathbf{u}^{H}(k+\tau)\right]^{\left(\frac{p}{2}\right)} e^{-j 2 \pi \tau k}\right\rangle_{k} \\
& =\left[R_{x, u_{j}}^{(p)}(\varepsilon ; \tau)\right]=\left\langle\mathbf{R}_{\mathrm{xu}}^{(p)}(\tau) e^{-j 2 \pi k\rangle_{t}}\right.
\end{aligned}
$$

where $\varepsilon$ represents the cycle frequency, $\tau$ represents the time delay, the element of ith row and jth column in the matrix is:

$$
R_{x_{i} u_{j}}^{(p)}(\varepsilon ; \tau)=\left\langle\mathbf{x}_{i}(k)^{\langle p\rangle}\left[\mathbf{u}_{j}^{H}(k+\tau)\right]^{\langle p\rangle} e^{-j 2 \pi \tau k}\right\rangle_{k}
$$

From formula(5),we can know when $0<p<\alpha,\left|R_{x u}^{(p)}(\tau)\right|<\infty$. Because of $\left|e^{-j 2 \pi k k}\right|=1,\left|R_{x u}^{(p)}(\varepsilon ; \tau)\right|<\infty$ Therefore, we can rewrite the formula (3) by using equation (15) and equation (17) :

$$
\max _{\mathbf{w}, \mathbf{c}} \frac{\left|\mathbf{w}^{H} \mathbf{R}_{\mathrm{xu}}^{(p)}(\varepsilon, \tau) \mathbf{c}\right|^{2}}{\mathbf{w}^{H} \mathbf{R}^{(p)}{ }_{\mathbf{x x}} \mathbf{w} \mathbf{C}^{H} \mathbf{R}^{(p)}{ }_{\mathbf{u u}} \mathbf{C}}
$$

where $\mathbf{u}(k)=\mathbf{x}^{*}(k+\tau)$ is the new referent signal.

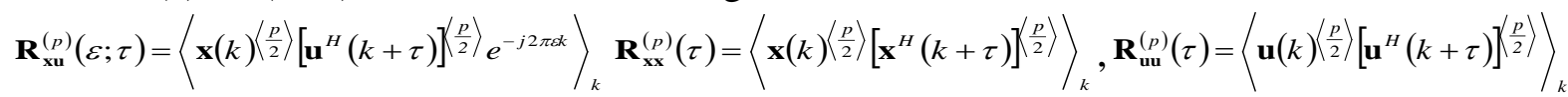

$\mathbf{R}_{\mathrm{xu}}^{(p)}(\varepsilon ; \tau)$ is the fractional lower order cyclic (FLOC) conjugate auto-correlation matrices about $\mathbf{x}(k)$. In addition, the $\mathbf{R}_{\mathbf{x x}}^{(p)}$ is the fractional lower order covariance(FLOC) about $\mathbf{x}(k) . \mathbf{R}_{\mathbf{u m}}^{(p)}=\mathbf{R}_{\mathbf{x}^{*} \mathbf{x}^{*}}^{(p)}$ is the same matrix about $\mathbf{x}^{*}(k)$.

It can easily be proved that equation (19) is equivalent to the following:

$$
\min _{\mathbf{w}, \mathbf{c}}\left\{\mathbf{w}^{H} \mathbf{R}_{\mathrm{xx}}^{(p)} \mathbf{w}+\mathbf{c}^{H} \mathbf{R}_{\mathrm{uu}}^{(p)} \mathbf{c}\right\} \text { subject to } \mathbf{w}^{H} \mathbf{R}_{\mathrm{xu}}^{(p)}(\varepsilon ; \tau) \mathbf{c c} \mathbf{c}^{H} \mathbf{R}_{\mathrm{xu}}^{(p)} \mathbf{w}=1
$$

where $\gamma$ is Lagrange multiplier parameters. We can set $\nabla_{\mathbf{w}} J(\mathbf{w}, \mathbf{c})=0, \nabla_{\mathbf{c}} J(\mathbf{w}, \mathbf{c})=0$ and minimize the cost function $J(\mathbf{w}, \mathbf{c})$ :

$$
\begin{gathered}
\mathbf{R}_{\mathbf{x x}}^{(p)}(\tau) \mathbf{w}=\gamma \mathbf{R}_{\mathbf{x u}}^{(p)}(\varepsilon ; \tau) \mathbf{c c}^{H} \mathbf{R}_{\mathbf{x u}}^{(p)^{H}}(\varepsilon ; \tau) \mathbf{w} \\
\mathbf{R}_{\mathbf{u u}}^{(p)}(\tau) \mathbf{c}=\gamma \mathbf{R}_{\mathbf{x u}}^{(p)}(\varepsilon ; \tau) \mathbf{w} \mathbf{w}^{H} \mathbf{R}_{\mathbf{x u}}^{(p)^{H}}(\varepsilon ; \tau) \mathbf{w}
\end{gathered}
$$

Formula (21) can be further deduced:

$$
\mathbf{c}=\gamma \mathbf{R}_{\mathbf{u u}}^{(p)^{-1}}(\tau) \mathbf{R}_{\mathbf{x u}}^{(p)^{H}}(\varepsilon ; \tau) \mathbf{w} \mathbf{W}^{H} \mathbf{R}_{\mathbf{x u}}^{(p)^{H}}(\varepsilon ; \tau) \mathbf{c}
$$

Substituting (22) into (21), gives:

$$
\mathbf{R}_{\mathbf{x x}}^{(p)}(\tau) \mathbf{w}=\xi \mathbf{R}_{\mathbf{x u}}^{(p)}(\varepsilon ; \tau) \mathbf{R}_{\mathbf{u u}}^{(p)^{-1}}(\tau) \mathbf{R}_{\mathbf{x u}}^{(p)^{H}}(\varepsilon ; \tau) \mathbf{w}
$$


From (23), weight vector $\mathbf{w}$ can be calculated as the eigenvector corresponding to the largest singular values of the following formula:

$$
\mathbf{R}_{\mathbf{x x}}^{(p)-1}(\tau) \mathbf{R}_{\mathbf{x u}}^{(p)}(\varepsilon ; \tau) \mathbf{R}_{\mathbf{u u}}^{(p)-1}(\tau) \mathbf{R}_{\mathbf{x u}}^{(p) H}(\varepsilon ; \tau)
$$

\section{Simulation Results}

A uniform linear array containing 10 half-wavelength spaced sensors is considered in the simulation. Without considering the effects of multipath, the direction of arrival of one desired signal and three interferences are $0^{\circ}$ and $-30^{\circ}, 30^{\circ}, 70^{\circ}$, respectively. The signal-to-interference ratio (SIR) is set to $-20 \mathrm{~dB}$ and the signal-to-noise ratio (SNR) is set to be $10 \mathrm{~dB}$. Besides, both desired signal and interference are modulated with binary-phase-shift-keying owning half cosine pulse shape. The sampling frequency is set to 1 . The baud rates for the desired signal and interference are $1 / 5,1 / 7,1 / 8$, and $1 / 9$ respectively while they share the same carrier frequency 0.25 . In this case, $\tau=0$ and $\alpha=1 / 5$. In the first three pieces of the figures, the number of sampling snapshots is 1500 . It is assumed that the power of desired signal is 1 . Hence, the power of noise and interference can be calculated by:

$$
\gamma=\sigma_{n}=10^{-\frac{S N R}{10}}, \sigma_{i}=10^{-\frac{S I R}{10}}
$$

If $\alpha$ stable noise exist in the receiving signal, $p=1.2, \alpha=1.6, \gamma=1$, other parameters are set to 0.

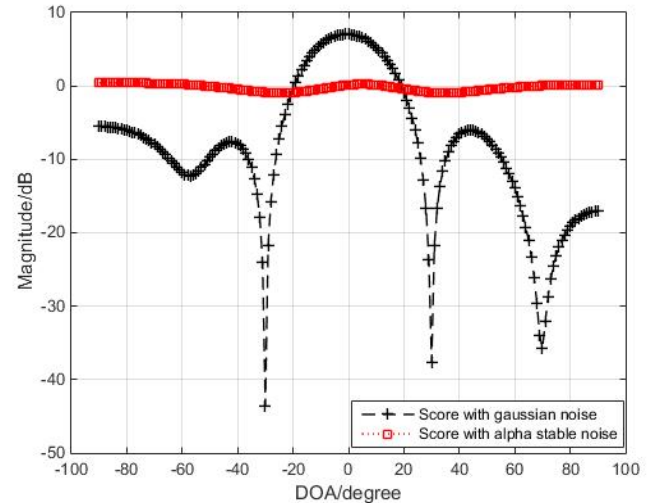

Fig. 1 beam patterns for traditional SCORE

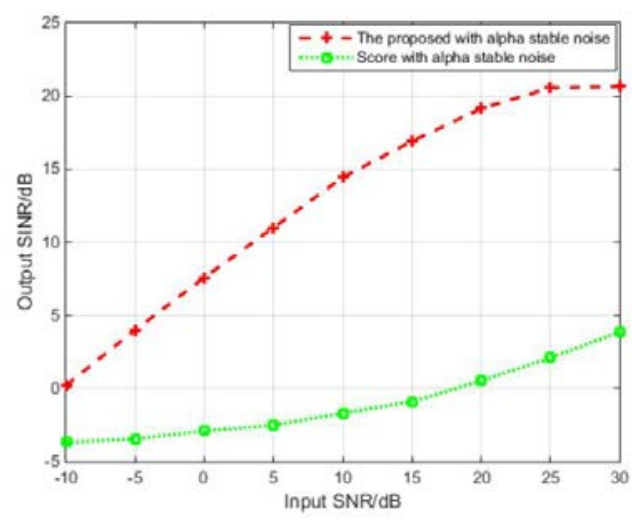

Fig. 3 SINR versus SNRs

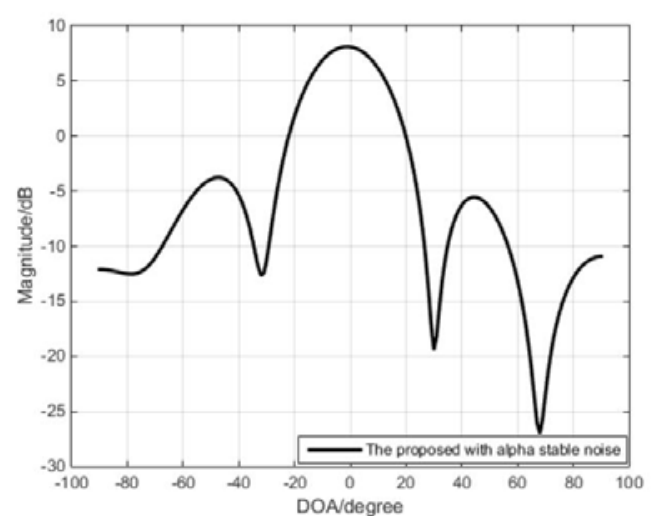

Fig. 2 beam patterns for the proposed

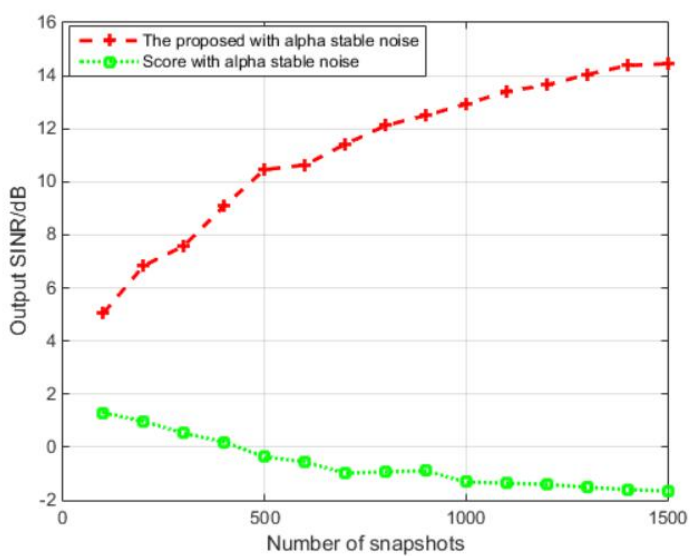

Fig. 4 SINR versus the number of snapshots

Fig. 1 illustrates the comparisons of the normalized beam patterns for traditional SCORE algorithm between gaussian white noise and $\alpha$ stable noise. It can be seen that in gaussian white noise environment, the traditional method will achieve an excellent performance while in $\alpha$ stable noise environment, it loses the ability to restore the desired signal and restrain the interference.

Fig.2 shows the simulation results of the proposed method through the employment of fractional lower order matrix in $\alpha$ stable noise environment. The proposed method not only provides a mean of 
interference suppression by forming deeper nulls in the direction of interference, but also achieves the highest array gains in the direction of desired signal.

In Fig.3 and Fig.4, we assume there are two interferences with sir=-20dB. The former presents the SINRs with different SNRs for $K=1500$. It is observed that the proposed method performs much better than the traditional method in $\alpha$ stable noise environment. Despite of the low SNR condition, the proposed method performs slightly worse due to the influence of the algorithm itself, while in comparison, the proposed method perform better in higher SNR with the increasing of SNR. Fig.4 shows the effect of the number of snapshots on the SINRs, from which it can be seen that the SINR obtained with the proposed method is much higher than the other with $\alpha$ stable noise.

\section{Conclusions}

In terms of the conventional SCORE algorithm, second-order matrix has been utilized to suppress interference and noise. However, if the characteristic index about signal or noise satisfies $0<\alpha<2$, such as $\alpha$ stable noise, there will not be the existence of second-order statistics or higher-order statistics.In this case, the conventional SCORE algorithm may suffer sharp deterioration in performance. To maintain the performance of SCORE algorithm, this paper proposes a method on the basis of robust estimation theory and fractional lower order matrix. Compared with other methods[7-8], the proposed method improve the robustness of the SCORE algorithm and is suitable for strong impulse noise, the correctness of which has been proved by the simulating results.

\section{Acknowledgements}

This work was supported by the Open Research Fund of State Key Laboratory of Space-Ground Integrated Information Technology under grant No.2014_CXJJ-DH_09, the National Natural Science Foundation of China (61201275), the Fundamental Research Funds for the Central Universities (ZYGX2013J026), the Guangdong Provincial Natural Science Foundation of china (2015A030313821).

\section{References}

[1] J. Capon, "High resolution frequency-wavenumber spectrum analysis,” Proc. IEEE, vol. 57, pp. 1408-1418, 1969.

[2] Taleb A,Jutten C.Blind Source Separation in Post-Nonlinear Mixtures.IEEE Transacitions on Signal Processing,1999, 47(10):2807-2820.

[3] E. Brennan, J. D. Mallet, and I. S. Reed, "Adaptive arrays in airborne MTI radar,"IEEE Trans. Antennas and Propagation, vol.24, no.5, pp.607-615, Sept. 1976.

$\begin{array}{ccccc}\text { [4] Javad, } & \text { Nedda } & \text { and } & \text { Ashjaee. } & \text { A }\end{array}$ Tutorial[DB/OL]. www.topconps.com/gpstutorial/TOC.html,1998.10.24.

[5] E. Y. Gorodetskaya, A. I. Malekhanov, A. G. Sazontov, and N. K. Vdovicheva, "Deep-water acoustic coherence at long ranges: theoretical prediction and effects on large-array signal processing," IEEE $J$ Ocean. Eng., vol.24, no.2, pp.156-171, Apr. 1999.

[6] H.L.V. TREES, Detection, Estimation and Modulation Theory. Part IV, Optimum Array Processing. New York: Wiley,2002.

[7] B. Kannan and W.J.Fitzgerald, "Beamforming in additive $\alpha$-stable noise using fractional lower statistics(FLOS)," IEEE International Conference on Electronics, Circuits and Systems, vol. 3,pp.1755-1758.

[8] P. Tsakalides and C.L. Nikias,'”Robust space-time adaptive processing(STAP) in non-Gaussian clutter environments,"IEE proceedings Radar, Sonar and Navigation, vol. 146, no.2, pp.84-93, April 1999. 
